\title{
Caracterização de culturas de urina realizadas no laboratório de análises clínicas do hospital universitário de Santa Maria - Santa Maria, RS, no \\ período de 2007 a 2010
}

\author{
Rosiéli Martini*, Rosmari Horner**, Magda Cristina Marques Souza \\ Roehrs*, Lívia Gindri***, Tassiane Paz Mielke***, Mônica de Abreu \\ Rodrigues*, Maísa Kraulich Tizotti*, Cláudia Barbisan Kempfer*, \\ Silvana Oliveira dos Santos ${ }^{\star \star *}$, Liliana Urdangarin de Sousa***, \\ Taíse Foletto****
}

RESUMO: A infecção do trato urinário (ITU) é considerada uma das patologias de origem bacteriana mais comum. $\mathrm{O}$ objetivo deste trabalho foi determinar a prevalência de ITU em pacientes atendidos no Hospital Universitário de Santa Maria (HUSM), o agente etiológico mais prevalente, a distribuição entre os sexos e a faixa etária mais acometida. Realizou-se um estudo retrospectivo das uroculturas solicitadas no HUSM, no período de 2007 a 2010. Dos 34.898 exames solicitados, $6.190(17,7 \%)$ apresentaram a identificação de algum agente bacteriano na amostra, sendo a Escherichia coli 0 microorganismo mais prevalente $(31,9 \%)$, além disso, observou-se um número significativo de leveduras do gênero Candida sp. (10,5\%), que ocupou o segundo lugar em relação aos outros agentes bacterianos. Em relação ao gênero dos pacientes, o sexo feminino apresentou a maior prevalência de culturas positivas (73\%) com distribuição principalmente na faixa etária entre 14 a 40 anos (37,6\%), e 0 masculino (27\%) prevalecendo na faixa entre 41 a 64 anos (10,6\%). A partir deste estudo pôde-se verificar que os bacilos gram-negativos (BGN) foram os patógenos prevalentes, além disso, ocorreu um significativo número de isolados de Candida sp.

Descritores: Infecção do trato urinário; Urocultura; Bacilos gram-negativos; Escherichia coli.

\section{Characterization of urine cultures performed in the clinical analysis laboratory of the university hospital of Santa Maria - Santa Maria, RS, the period 2007 to 2010}

ABSTRACT: A urinary tract infection (UTI) is considered one of the diseases most common bacterial. The objective of this study was to determine the prevalence of UTI in patients treated at University Hospital of Santa Maria (HUSM), the most prevalent agent, distribution between sexes and age groups most affected. We conducted a retrospective study of urine cultures HUSM requested in the period 2007 to 2010 . Of the 34,898 exams, $6,190(17.7 \%)$ presented the identification of a bacterial agent in the sample. The most prevalent organism was Escherichia coli with $31.9 \%$, also observed a significant number of yeasts Candida sp. (10.5\%), which ranked second in relation to other bacterial agents. Regarding the gender of the patients, females had the highest prevalence of positive cultures $(73 \%)$ with distribution primarily in the age group 14 to 40 years $(37.6 \%)$ and male $(27 \%)$ prevailing in the range from 41 to 64 years (10.6\%). From this study it was observed that Gram-negative bacilli (GNB) were the prevalent pathogens, in addition, there was a significant number of isolates of Candida sp.

Descriptors: Urinary tract infection; Urine culture; Gram-negative; Escherichia coli.

\footnotetext{
* Mestranda do Programa de Pós-Graduação em Ciências Farmacêuticas, Universidade Federal de Santa Maria, Santa Maria, Rio Grande do Sul, Brasil.

** Professora Adjunta do Departamento de Análises Clínicas e Toxicológicas, Universidade Federal de Santa Maria, Santa Maria, Rio Grande do Sul, Brasil.

*** Farmacêuticas graduadas pela Universidade Federal de Santa Maria, Santa Maria, Rio Grande do Sul, Brasil.

**** Aluna do Curso de Graduação em Farmácia, Universidade Federal de Santa Maria, Santa Maria, Rio Grande do Sul, Brasil.
} 


\section{Introdução}

A Infecção do Trato Urinário (ITU) é caracterizada pela presença de microorganismos (normalmente bactérias) que se multiplicam nas vias urinárias, podendo ser uma doença sintomática ou assintomática, com ocorrência frequente e que pode acometer pessoas de qualquer faixa etária, hospitalizada ou não. ${ }^{1}$ As ITUs são as infecções bacterianas mais comuns, dados demonstram que elas representam aproximadamente 150 milhões de casos por ano em todo o mundo, ${ }^{2}$ sendo considerada a segunda infecção mais prevalente no ser humano. ${ }^{3}$ Nos Estados Unidos, cerca de 3 a $4 \%$ das consultas médicas anuais em mulheres estão ligadas à sintomas de ITU, ${ }^{4}$ sendo que no Brasil esse índice é maior, correspondendo a $8 \% .^{5}$

A ITU pode comprometer somente o trato urinário inferior, manifestando-se como cistite, ou afetar concomitantemente o trato urinário inferior e o superior, caracterizando uma nefrite. ${ }^{6}$ O diagnóstico de uma ITU sintomática pode ser sugerido pelo quadro clínico que pode incluir: disúria, polaciúria, dor lombar e/ou supra púbica, febre e calafrios, urgência miccional, nictúria, urina turva e/ou avermelhada, mas podendo somente ser confirmado com a realização da urocultura, teste considerado o padrão-ouro para confirmação do quadro. ${ }^{7}$

A realização da urocultura não só indica a presença de bactérias na urina, mas também permite isolar o agente causador da infecção e a execução do antibiograma, o qual determina a sensibilidade do microorganismo frente aos antibióticos permitindo estabelecer o tratamento correto. ${ }^{8}$ Uma cultura de urina é considerada positiva quando a contagem das Unidades Formadoras de Colônia por mililitro (UFC/mL) de urina for igual ou superior a 100.000 ( $\geq 10^{5}$ UFC/ml) em amostra coletada a partir do jato médio, e de maneira asséptica. ${ }^{9}$ Porém, nas infecções crônicas, em pacientes idosos ou em uso vigente de antimicrobianos, a contagem de $10^{4} \mathrm{UFC} / \mathrm{mL}$ deve ser considerado positiva. ${ }^{10}$

No que se refere à suscetibilidade da infecção entre os gêneros, essa varia conforme a faixa etária, sendo que no primeiro ano de vida é mais frequente no sexo masculino devido às más formações congênitas. ${ }^{11}$ Após essa idade, o sexo feminino é o mais acometido: devendose às condições anatômicas da genitália feminina, na qual a uretra é mais curta e mais próxima do ânus. ${ }^{3}$ Existem ainda vários outros fatores que favorecem a ITU nas mulheres, dentre eles, episódios anteriores de cistite, relação sexual, gestação e a higiene precária.,10

Já em relação ao adulto do sexo masculino, os fatores de risco para uma ITU incluem, principalmente, a instrumentação das vias urinárias (cateterismo vesical) e a hiperplasia da próstata. ${ }^{12,13}$ Nos idosos, com idade superior a 65 anos, o índice de ITU entre os sexos não apresenta grandes oscilações. ${ }^{14} \mathrm{~A}$ idade, a presença de doenças de base, lesões na medula espinhal e 0 uso de instrumento como 0 cateterismo urinário são fatores desencadeantes de ITU. ${ }^{15,16}$

Diversos tipos de microorganismos podem causar ITU incluindo protozoários, fungos, vírus e bactérias, sendo que estes últimos são a principal causa de infecções urinárias, responsável por mais de $95 \%$ dos casos. ${ }^{17}$ As principais bactérias envolvidas nas ITUs são as da família Enterobacteriaceae, em especial a Escherichia coli, bem como cocos grampositivos, bacilos gram-negativos não-fermentadores da glicose como a Pseudomonas aeruginosa além de fungos como a Candida albicans. ${ }^{18}$

A E. coli é a bactéria mais isolada em ITUs, ${ }^{19}$ constituindo 0 principal agente das infecções urinárias adquiridas na comunidade, na faixa de 70 a $85 \%$ dos casos, seguida de 0 Staphylococcus saprophyticus, Proteus sp., Klebsiella sp. e Enterococcus faecalis. ${ }^{6}$ Já no 
âmbito hospitalar divide a predominância com outras bactérias como Klebsiella sp., Proteus sp., Pseudomonas sp., Enterobacter sp., e com gram-positivos, como Enterococcus sp. e Staphylococcus sp.. ${ }^{5}$ Pseudomonas sp. e Proteus sp. são as bactérias mais frequentemente isoladas de infecções polimicrobianas..$^{20,21}$

Dentre as infecções nosocomiais as ITUs são responsáveis por 40 a 50\% dos casos. ${ }^{22}$ Esta frequência elevada de ITUs no ambiente hospitalar nos estimulou a efetuar este estudo retrospectivo, para avaliar a prevalência de uroculturas positivas provenientes de pacientes atendidos no Hospital Universitário de Santa Maria (HUSM) e identificar o agente mais isolado.

\section{Material e métodos}

\section{Estudo retrospectivo}

Realizou-se um estudo transversal retrospectivo da análise dos resultados de todas as uroculturas solicitadas durante os anos de 2007 a 2010, de pacientes com idade igual ou superior a 1 ano, atendidos no HUSM de Santa Maria, Rio Grande do Sul.

As urinas foram processadas de acordo com o Procedimento Operacional Padrão (POP) do Laboratório de Análises Clínicas (LAC) do HUSM. As amostras foram semeadas pelo método da alça calibrada, cultura quantitativa, no qual, um volume de 0,01 $\mathrm{mL}(10 \mu \mathrm{L})$ era semeado em ágar CLED (Cystine Lactose-Electrolyte-Deficient), ágar MacConkey (MC), e ágar sangue, quando necessário, também é feito o exame bacterioscópico. Na presença de $\geq 10^{5} \mathrm{UFC} / \mathrm{mL}$ as amostras foram consideradas positivas para bactérias. Após isolamento dos microorganismos, realizaram-se testes de identificação e avaliou-se o perfil de sensibilidade aos antimicrobianos através da automação (MicroScan® - Siemens).

Quando da observação de blastoconídeos e hifas no Gram nas amostras de urina, analisaram-se os resultados dos Exames Qualitativos de Urina (EQU) a fim de verificar se foi reportada a presença desses elementos. Após 24 horas, se no meio de CLED ocorreu 0 crescimento de colônias brancas, fez-se um novo Gram com esta colônia e, se neste fora visualizado blastoconídeos ou hifas, realizaram-se a contagem dessas colônias. No caso de contagem superior a $40.000 \mathrm{UFC} / \mathrm{mL}$, encaminharam-se essas amostras para 0 setor de Micologia do HUSM a fim de identificar a espécie e, a critério médico a realização do fungigrama.

O trabalho foi aprovado pelo Comitê de Ética em Pesquisa (CEP) da Universidade Federal de Santa Maria (UFSM), sob o número 0235.0.243.000-08.

\section{Resultados}

Foram avaliadas 34.898 uroculturas no período de janeiro de 2007 a dezembro de 2010, sendo que um total de 6.190 destas $(17,7 \%)$ amostras apresentou resultado positivo.

Das amostras positivas 4.515 (73\%) eram do sexo feminino, e $1.675(27 \%)$ do sexo masculino. A distribuição das uroculturas positivas entre os gêneros está mostrada na figura I. 


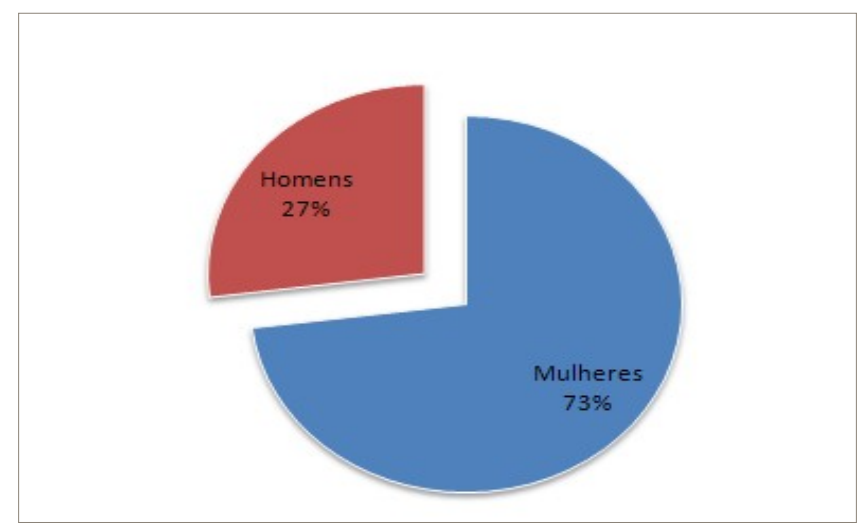

Figura I - Distribuição das uroculturas positivas de acordo com o gênero dos pacientes no período de 2007 a 2010 no HUSM, SM, RS.

Entre as mulheres, a faixa etária mais prevalente foi de 14 a 40 anos (37,6\%), já nos homens a de 41 a 64 anos (10,6\%), o que pode ser observado na Tabela 1.

Tabela 1 - Distribuição percentual das uroculturas positivas nas diferentes faixas etárias.

\begin{tabular}{cccccc}
\hline & \multicolumn{1}{c}{ Sexo } & \multicolumn{3}{c}{ Faixa Etária } \\
& & 1-13 anos & $14-40$ anos & $41-64$ anos & $\geq 65$ anos \\
\hline \multirow{2}{*}{ Percentual } & Feminino & $5,3 \%$ & $37,6 \%$ & $17 \%$ & $11,3 \%$ \\
& Masculino & $1,6 \%$ & $5,5 \%$ & $10,6 \%$ & $9,6 \%$ \\
\hline
\end{tabular}

Aproximadamente $65 \%$ das bactérias isoladas neste estudo foram gram-negativas. A prevalência dos agentes etiológicos nas uroculturas do HUSM encontra-se na figura 2.

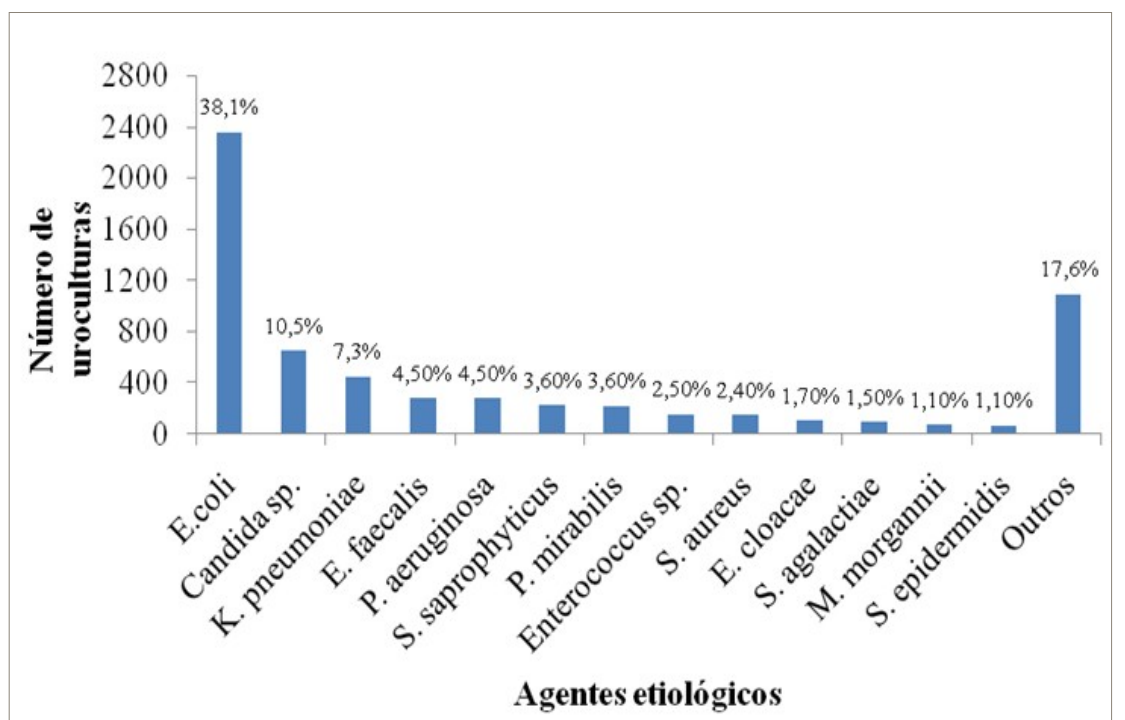

Saúde (Santa Maria), v.37, n.1, p. 55-64,

Caracterização de culturas de uina r niversitário de Santa Maria - Santa Maria, RS, no periodo de 2007 a 2010 ISSN 2236-5834

Figura 2 - Prevalência dos agentes etiológicos das uroculturas positivas no período de 2007 a 2010 no HUSM, SM, RS.

Escherichia coli $=$ E. coli; Klebsiella pneumoniae $=$ K. pneumoniae; Enterococcus faecalis $=$ E. faecalis; Pseudomonas aeruginosa = P. aeruginosa; Staphylococcus saprophyticus $=\mathrm{S}$. saprophyticus; Proteus mirabilis $=$ P. mirabilis; Staphylococcus aureus $=\mathrm{S}$. aureus; Enterobacter cloacae $=$ E. cloacae; Streptococcus agalactiae $=\mathrm{S}$. agalactiae; Morganella morgannii $=\mathrm{M}$. morgannii; Staphylococcus epidermidis $=\mathrm{S}$. epidermidis. Outros $=$ Acinetobacter baumanni, Serratia marcescens, Staphylococcus haemolitycus, Enterococcus faecium. 
Das culturas positivas foram 97\% monomicrobianas e 3\% (187 de 6.190) polimicrobianas, sendo que a $\mathrm{E}$. coli foi a bactéria prevalente nas culturas polimicrobianas, 53 de 187 casos, perfazendo $28,3 \%$.

\section{Discussão}

As ITUs são as infecções bacterianas mais comuns relatadas tanto no âmbito hospitalar quanto na comunidade. ${ }^{23}$ Essas podem ocorrer em qualquer fase da vida. Dados demonstram que cerca de 7 milhões de americanos, principalmente mulheres, buscam auxílio médico decorrente de sintomas típicos de ITUs. ${ }^{24}$ As uroculturas constituem um dos exames clínicos mais requisitados em laboratórios de microbiologia clínica. ${ }^{25}$

No que se refere aos índices de positividade de uroculturas, esses variam muito conforme a região. Em nosso estudo, a taxa de positividade foi de 17,7\%. Esse dado é semelhante a outros estudos realizados no Brasil. Ramos e cols, em 2006 (São Paulo), encontraram 15\%; Hörner e cols, em 2008 (Santa Maria), 16,4\% e Muller e cols, em 2008 (Paraná), 15,9\%.26-28 Porém, no norte e nordeste do Brasil essa prevalência foi maior. Vieira e cols, em 2007 (Pará) e Costa e cols, em 2010 (Paraíba) relataram 22,6\% e 22,8\%, respectivamente. ${ }^{29,30}$

Entre os fatores que predispõem a maior frequência de infecções urinárias nas mulheres, destaca-se a anatomia genitourinária feminina. ${ }^{3}$ Dessa forma, ratificam-se os dados obtidos em nosso estudo, o qual demonstrou que mais de $70 \%$ das uroculturas positivas foram provenientes de mulheres, concordando com os demais estudos descritos na literatura. ${ }^{26,28-32}$

Em relação às faixas etárias determinadas neste estudo, tratando-se do sexo feminino, a idade mais acometida foi entre 14 a 40 anos (37,6\%). Esse intervalo corresponde à fase fértil da mulher, a qual normalmente se encontra em atividade sexual, e mais propícia às ITUs. ${ }^{5}$ Outra pesquisa efetuada com população adulta, no Paraná, também mostrou que nesta faixa etária ocorreu a maior percentual de ITU $(38,5 \%) .{ }^{28}$ No entanto, com o sexo masculino, a idade prevalente no nosso estudo foi superior à 41 anos $(20,2 \%)$, concordando com o relato de Muller e cols. ${ }^{28}$

As bactérias prevalentes nas ITUs são as gram-negativas, especialmente a E. coli. Isso se deve ao fato das mesmas serem colonizadoras da flora intestinal e eventualmente poderem causar infecções extra-intestinais. ${ }^{33}$ Dentre essas bactérias destaca-se a E. coli. ${ }^{19}$ corroborando com nossos achados, no qual ela foi responsável por 38,1\%. A E. coli extraintestinal, "Uropathogenic E.coli" (UPEC) causa frequentemente ITUs, devido ao fato de possuir fatores de virulência como as fímbrias do tipo 1 e $P$, que se aderem nas células da uretra e iniciam a infecção. ${ }^{34}$

Toda a família Enterobacteriacea destaca-se significativamente na epidemiologia das ITUs, ${ }^{35}$ além da E.coli, K. pneumoniae foi a segunda bactéria mais isolada, esse dado está em concordância com outros relatos na literatura, como por exemplo, em Salvador, e também em estudos fora do Brasil, em Madagascar e na Jordânia. ${ }^{31,36-38}$ Além disso, neste estudo, bactérias gram-negativas não-fermentadoras também foram isoladas, com destaque para a $P$. aeruginosa, oportunista nosocomial relevante, que se torna patogênico em indivíduos com 0 com o sistema imune debilitado e possui um elevado índice de resistência frente aos antimicrobianos. ${ }^{39,40}$

Saúde (Santa Maria), v.37, n.1, p. 55-64, 2011.

As bactérias gram-positivas, em nosso estudo, corresponderam a aproximadamente $25 \%$ Martini, R. etal. das identificações. Entre essas bactérias, o E. faecalis, foi o agente mais prevalente ocupando 
o quarto lugar entre os patógenos mais isolados nas ITUs, corroborando com Setteh, em $2004 .{ }^{37}$ O S. saprophyticus foi responsável por $3,6 \%$ das uroculturas positivas. Dados da literatura demonstram que esse microorganismo vem emergindo como causador de ITUs, ${ }^{5}$ sendo isolado frequentemente em mulheres com atividade sexual na faixa etária entre 13 a 40 anos. ${ }^{41}$

Além de bactérias, foi identificado um número significativo de fungos do gênero Candida sp., (10,5\%) nas uroculturas realizadas no HUSM, concordando com outros estudos. ${ }^{42,43} \mathrm{~A}$ Candida albicans é frequentemente identificada em culturas de urina, principalmente de pacientes imunocomprometidos. ${ }^{18}$

Pseudomonas sp. $(16,4 \%)$ e Proteus sp. $(9,09 \%)$ foram as bactérias mais frequentes nas uroculturas polimicrobianas em nosso estudo, o que concorda com a literatura. ${ }^{20,21}$

Comparando o presente estudo de quatro anos com outro estudo realizado em 2008, por Hörner e cols., na mesma instituição, concluímos que ocorreu uma relevante modificação na prevalência dos agentes isolados nas uroculturas provenientes dos pacientes atendidos no HUSM. Estudo este, identificou a P. aeruginosa, E. cloacae e S. aureus como segundo, quinto e sexto patógenos mais isolados. ${ }^{44}$ No entanto, a E. coli se manteve como 0 agente mais isolado nas ITUs numa proporção menor.

Além disso, podemos destacar um significativo número de isolados de Candida sp., microorganismo que ocupou o segundo lugar em relação aos outros agentes etiológicos nas ITUs. Isto deve ser criteriosamente avaliado, pois 0 isolamento de Candida sp. em urinas pode despertar dúvidas em relação a sua interpretação. Visto que, pode corresponder a uma contaminação no momento da coleta de urina ou à colonização, bem como, uma ITU assintomática, cistite ou pielonefrite, candidíase renal primária ou candidíase disseminada com manifestação renal. ${ }^{45}$ Estudos transversais de prevalência devem ser estimulados, a fim de se determinar a flora microbiana prevalente em cada instituição ou região do país.

\section{Agradecimentos}

À equipe do Laboratório de Análises Clínicas (LAC) do Hospital Universitário de Santa Maria (HUSM) / RS.

\section{Conflito de interesses}

Não houve nenhum tipo de conflito de interesse no desenvolvimento deste estudo.

\section{Suporte Financeiro}

Programa de Pós-Graduação em Ciências Farmacêuticas da Universidade Federal de Santa Maria (PPGCF / UFSM) e a Coordenação de Aperfeiçoamento de Pessoal de Nível Superior (CAPES).

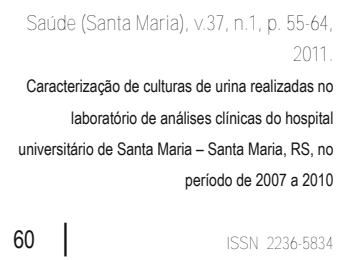




\section{Referências}

1. Duarte G. Infecção Urinária durante a gravidez. Rev Bras de Ginec e Obst 1997;19: 495-503.

2. Tortora GJ; Funke BR, Case CL. Microbiologia. Traduzido por Roberto Marchiori Martins. $8^{\text {a }}$ ed. Porto Alegre: Artmed, 2005.

3. Valiquette L. Urinary tract infections in women. Can J Urol 2001;8: 6-12.

4. Nicolle, L. E. Epidemiology of urinary tract infections. Infect Med 2001:18:153- 162.

5. Dalbosco V, Srougi M, Dall'Oglio M. Infecções do Trato Urinário. Rev Bras Med 2003;60(6):320-336.

6. Hooton TM, Stamm WE. Diagnosis and treatment of uncomplicated urinary tract infection. Infect Dis Clin North Am 1997;11:551-81.

7. Santos SRSR, Amado CAB, Assef SMC. Infecções urinárias. Arquivos de Ciências da Saúde Unipar 1999;3:43-50.

8. Rossi F, Andreazzi DB. Resistência bacteriana: interpretando o antibiograma. Editora Atheneu, São Paulo, 2005.

9. Kass EH. Assintomatic infections of the urinary tract. Trans Ass Am Phys 1956;69:56-63.

10. Fihn SD. Clinical practice. Acute uncomplicated urinary tract infection in women. $\mathrm{N}$ Engl J Med 2003;349:259-66.

11. Vieira Neto OM. Infecção do Trato Urinário. Med 2003;36:365-9.

12. Pewitt EB, Schaeffer AJ. Urinary tract infection in urology, including acute and chronic prostatitis. Infect Dis Clin North Am 1997;11:623-46.

13. Warren JW. Catheter-associated urinary tract infections. Infect Dis Clin North Am 1997;11:609-22.

14. Agência Nacional da Vigilância Sanitária - ANVISA. Manual de Microbiologia Clínica para o Controle de Infecção em Serviços de Saúde - Principais Síndromes Infecciosas: Módulo I - Infecções do trato urinário. $1^{\text {a }}$ ed. Edição Comemorativa para o IX Congresso Brasileiro de Controle de Infecção e Epidemiologia Hospitalar, Salvador, 2004. Disponível em: http://www.ccih.med.br/mod_1_2004.pdf. Acessado em junho de 2011.

15. Ruscadella FF, Elía S, Sánchez EG, Rodríguez, JAG. Estudio de prevalencia de la infección urinaria en la comunidad y su sensibilidad a la cefixima. Rev Esp Quimioterap 1999;12:41-47.

16. Goldstein FW, Multicentre Study Group. Antibiotic susceptibility of bacterial strains isolated from patients with community-acquired urinary tract infections in France. Eur J Clin Microbiol Infect Dis 2000;19:112-117.

17. Bonadio M, Meini M, Spitaler P, Gigli C. Current microbiological and clinical aspect of urinary tract infection. Eur Urol 2001; 40:439-445.

18. Souto CAV, Dias BS. Infecção do Trato Urinário por fungo. Intern Braz Journ of Urol 2003;29(3):56-9.

19. Farajnia S, Alikhani MY, Ghotaslou R, Naghili B, Nakhlband A. Causative agents and antimicrobial susceptibilities of urinary tract infections in the northwest of Iran. Int J Infect Dis 2009;13(2):140-144.

20. Ronald A. The etiology of urinary tract infection: Traditional and emerging pathogens. Am J Med 2002;113(1A):14-19.

21. Shortliffe LD, McCue JD. Urinary tract infection at the age extremes: Pediatrics and geriatrics. Am J Med 2002;113(1A):55S-66S.

22. Barnes RC, Daifuku R, Roddy RE, Stamm WE. Urinary-tract infection in sexually active homosexual men. Lancet 1986;:1:171-3. .

Saúde (Santa Maria), v.37, n.1, p. 55-64, 2011.

23. Sussman M. Urinary tract infections. In: Collier L, Balows A, Sussman M, eds. Topley and Wilson's Martini, R. etal. Microbiology and Microbial Infections, 9th edn. London: Arnold, 1998; 601-21. 
24. Vasquez Y, Hand WL. Antibiotic Susceptibility Patterns of Community-Acquired Urinary Tract Infection Isolates from Female Patients on the US (Texas)- Mexico Border. J Appl Res 2004;4(2);321.

25. Bail L, Ito CAS, Esmerino LA. Infecção do trato urinário: comparação entre o perfil de susceptibilidade e a terapia empírica com antimicrobianos. Rev Bras Anál Clín 2006;38(1):51-56.

26. Ramos TZ, Pizzolitto EL, Pizzolitto AC. Uso do teste com cloridrato de trifenil tetrazólio (CTT) para detecção de bacteriúria sintomática e assintomática. Rev Bras Anál Clín 2006;38(3):197-199.

27. Hörner R, Kocourek GED, DomingueVO, Rigatti F, Bertoncheli CM, Paraguinski GL. Comparação de métodos de triagem para detecção de bacteriúria em amostras do Bairro Maringá e do Hospital Universitário de Santa Maria. Saúde, Santa Maria 2008;34a,(1-2):16-21.

28. Muller EV, Santos DF, Corrêa NAB. Prevalência de microrganismos em infecções do trato urinário de pacientes atendidos no laboratório de análises clínicas da Universidade Paranaense - Umuarama - PR. Rev Bras Anál Clín 2008;40(1): 35-37.

29. Vieira JMS, Saraiva RMC, Mendonça LCV, Fernandes VO, Pinto MRC, Vieira ABR. Suscetibilidade antimicrobiana de bactérias isoladas de infecções do trato urinário de pacientes atendidos no Hospital Universitário Bettina Ferro de Souza, Belém-PA. Rev Bras Anál Clín 2007;39(2):119-121.

30. Costa LC, Belém LF, Silva PMF, Pereira HS, Silva Júnior ED, Leite TR et al. Infecções urinárias em pacientes ambulatoriais: prevalência e perfil de resistência aos antimicrobianos. Rev Bras Anál Clín 2010;42(3):175-180.

31. Randrianirina F, Soares JL, Carod JFC, Ratsima E, Thonnier V, Combe P et al. Antimicrobial resistance among uropathogens that cause community-acquired urinary tract infections in Antananarivo, Madagascar. J Antimicrob Chemother 2007;59:309-312.

32. Arjunan M, Salamah AAA, Amuthan M. Prevalence and Antibiotics Susceptibility of Uropathogens in Patients from a Rural Environment, Tamilnadu. Am J Infect Dis 2010;6(2):29-33.

33. Moura LB, Fernandes MG. A Incidência de Infecções Urinárias Causadas por E. coli. Revista Olhar Científico (Faculdades Associadas de Ariquemes) 2010:1(2):411-426.

34. Trabulsi LR, Alterthun F. Microbiologia. $4^{a}$ ed. e $5^{\mathrm{a}}$ ed. São Paulo: Atheneu, 2008.

35. Jawetz E, Levinson W. Microbiologia Medica e Imunologia. $7^{7 a}$ ed. Porto Alegre, Editora Artmed, 2005:632.

36. Sousa Jr MA, Fernandez LG. Perfil de Susceptibilidade aos Antimicrobianos mais Comercializados para o Tratamento de Infecções do Trato Urinário no Ano de 2003 em Salvador - BA. NewsLab 2004;67:96-106.

37. Abu-Setteh MH. Uropathogens and their susceptibility patterns at king hussein medical Center-Jordan. Gülhane Tıp Dergisi 2004;46(1):10-14.

38. Naeem M, Khan MA, Qazi SM. Antibiotic Susceptibility Pattern of Bacterial Pathogens Causing Urinary Tract Infection in a Tertiary Care Hospital. Ann Pak Inst Med Sci 2010;6(4): 214-218.

39. Inan D, Öğüç D, Günseren F, Çolak D, Mamikoğlu L, Gültekin M. The resistance of Pseudomonas aeruginosa strains isolated from nosocomial infections against various antibiotics. Bulletin of Microbiology 2000;34:255.

40. Menezes EA, Brasil JS, Rocha MVAP, Andrade MSS, Monteiro MNR, Cavalcante MS et al. Infecções urinárias por Pseudomonas aeruginosa em pacientes cateterizados na Clínica de Urologia da Santa Casa da Misericórdia de Fortaleza-Ceará. Rev Bras Anál Clín 2003;35(2):77-79.

41. Guidoni EBM, Toporovski J. Infecção urinária na adolescência. J Pediatria 2001;77(2):165-169.

Saúde (Santa Maria), v.37, n.1, p. 55-64,

Caracterização de culturas de urina realizadas no aboratório de análises clinicas do hospital universitário de Santa Maria - Santa Maria, RS, no periodo de 2007 a 2010

42. Nizami SQ, khan IA, Farooqui BI. Treatment of UTI in children in Karachi, Pakistan: Which antibiotic to use? Infect Dis J Pak 1997;25-6.

43. Ahmed A, Zafar A, Rizvi S. Quinolone resistance among common urinary tract pathogens E.coli and Klebsiella. Infect Dis J Pak 2000;7:12. 
44. Hörner R, Vissoto R, Mastella A, Salla A, Meneghetti B, Dal Forno NLF et al. Prevalência de microrganismos em infecções do trato urinário de pacientes atendidos no Hospital Universitário de Santa Maria. Rev Bras Anál Clín 2006;38(3): 147-150.

45. Gomes CL, Cavalcante JE, Cunha FA, Amorim LN, Menezes EA. Identificação e perfil de sensibilidade de Candida spp isoladas de urina de pacientes com Candidúria em Iguatu-Ceará. Rev Bras Anál Clín 2010; 42(3):223-225.

\section{Rosiéli Martini}

Endereço para correspondência - Rua Floriano Peixoto 1321 ap 202, Centro, Santa Maria, Rio Grande do Sul, Brasil.

Email: rosifarma@gmail.com

Recebido em 15 de julho de 2011.

Aprovado em 14 de setembro de 2011. 